\section{P293 THE POTENTIAL ROLE OF MASTURBATION IN TRANSMITTING NEISSERIA GONORRHOEAE AT MULTIPLE ANATOMICAL SITES AMONG MEN WHO HAVE SEX WITH MEN}

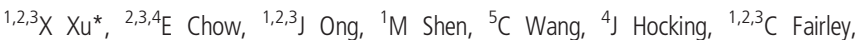
1,2,3,5 Z Zhang. 'China Australia Joint Research Center for Infectious Diseases, School of Public Health, Xi'an Jiaotong University Health Science Centre, Xi'an, People's Republic of China; ${ }^{2}$ Melbourne Sexual Health Centre, Alfred Health, Melbourne, Australia; ${ }^{3}$ Central Clinical School, Faculty of Medicine, Nursing and Health Sciences, Monash University, Melbourne, Australia; ${ }^{4}$ Centre for Epidemiology and Biostatistics, Melbourne School of Population and Global Health, The University of Melbourne, Melbourne, Australia; ${ }^{5}$ Department of Epidemiology and Biostatistics, College of Public Health, Zhengzhou University, Zhengzhou, People's Republic of China
\end{abstract}

\subsection{6/sextrans-2021-sti.355}

Background Neisseria gonorrhoeae can be cultured from saliva in men with pharyngeal gonorrhoea and could theoretically be transmitted from the pharynx to the urethra when saliva is used as a lubricant for masturbation. To explore this issue, we developed mathematical models for the transmission of Neisseria gonorrhoeae at each of oropharynx, urethra and anorectum among men who have sex with men (MSM).

Methods Model 1 included transmission routes (oral sex, anal sex, rimming, kissing, and three sequential sex practices) we have previously validated. In Model 2, we added masturbation to model 1. In Model 3, we included masturbation but excluded the three sequential sex practices. We calibrated our data to six international studies. We evaluated the model performance using the Root Mean Squared Error (RMSE) and Cohen's d statistic.

Results Model 2 has significantly higher RMSE than model 1 ( $p$-value $<0.01$ in five datasets, and $p=0.47$ in one dataset), but only $\mathrm{p}$-values from two datasets revealed a substantially large effect (Cohen's $d>0.8$ ) compared with Model 1. This suggests performance of Model 1 and Model 2 are similar. In contrast, Model 3 has significantly higher RMSE than both Model 1 and Model 2 (p-value $<0.01$ for all six datasets), and p-value revealed a large effect (Cohen's $\mathrm{d}>0.8$ for all six datasets) compared with the two models. This suggests performance of Model 3 is significantly worse than Model 1 and Model 2.

Conclusion Our findings indicate that masturbation plays a moderate role in the transmission of Neisseria gonorrhoeae. Our model also suggests that sequential sexual practices may be more important than masturbation for explaining the sitespecific prevalence in men with multi-site infection. Our model predicted that about 1 in 4 cases of urethral gonorrhoea might arise from masturbation if it transmits gonorrhoea.

\section{P296 DO GBMSM'S PREFERENCES FOR IN-PERSON, TELEPHONE OR DIGITAL SEXUAL HEALTHCARE VARY ACCORDING TO HEALTH CONCERNS AND SYMPTOMS? A CROSS-SECTIONAL SURVEY}

${ }^{1} \mathrm{R}$ Kincaid* ${ }^{1} \mathrm{C}$ Estcourt, 2J Gibbs, if Dalrymple, if Frankis, ${ }^{1}$ Glasgow Caledonian University, Glasgow, UK; ${ }^{2}$ University College London, London, UK

\subsection{6/sextrans-2021-sti.356}

Background As sexual healthcare moves online, it's important to understand the needs and preferences of groups with a higher burden of poor sexual health, to ensure equitable services. We explored gay, bisexual, and other men who have sex with men's [GBMSM] preferences for in-person, telephone, and online provision of sexual healthcare and whether preferences change in the presence of symptoms and/or concerns about STI risk.

Methods Cross-sectional online survey of GBMSM in Scotland recruited from sexual-social media 12/2019-03/2020 (preCovid-19 pandemic). Participants were asked their preferences (or no preference) for accessing appointment booking, providing sexual/medical history, and accessing HIV/STI results in two scenarios: routine check-up (no symptoms/concerns); and concerned about new symptoms/possible infection. Data were analysed using Pearson chi-squared, McNemar-Bowker, and post-hoc McNemar tests.

Results 755 GBMSM participated, median age 39, 71.4\% completed higher education, 69.9\% were White Scottish. When accessing a routine check-up, proportions preferring in person, telephone and online care respectively were: booking appointments [27/755 (3.6\%), 113/755 (15.0\%), 520/755 (68.9\%)]; reporting sexual behaviour [184/748 (24.6\%), 39/ 748 (5.2\%), 382/748 (51.1\%)]; reporting symptoms [254/747 (34.0\%), 46/747 (6.2\%), 308/747 (41.2\%)]; reporting medication $[163 / 745$ (21.9\%), 46/745 (6.2\%), 358/745 (48.1\%)]; receiving HIV results [200/699 (28.6\%), 73/699 (10.4\%), 304/ 699 (43.5\%)]; receiving STI results [143/746 (19.2\%), 96/746 (12.9\%), 361/746 (48.4\%)]. A significant proportion of participants' preferences changed across all elements of care measured, when concerned about symptoms or infection $(p<0.005)$. Post-hoc analyses suggest that these changes were mostly attributed to a shift in preference from online to inperson care in the presence of symptoms/STI risk.

Conclusions In this online-recruited sample of highly educated, older GBMSM, online care was highly acceptable but a significant proportion preferred in-person care in the presence of symptoms/STI risk. Choice in sexual healthcare provision is essential as GBMSM's preferences are not static and appear highly associated with emotional context.

\section{P298 ANTIBIOTIC USE AND CHLAMYDIA INFECTION AMONG WOMEN AT REPRODUCTIVE AGE IN GUANGDONG, CHINA}

${ }^{1,2} \mathrm{X}$ Sun*, ${ }^{1} \mathrm{C}$ Li, ${ }^{1} \mathrm{C}$ Wang, ${ }^{1} \mathrm{~B}$ Yang, ${ }^{1,2} \mathrm{~W}$ Tang. 'Department of Sexually Transmitted Disease Prevention and Control, Dermatology Hospital of Southern Medical University, Guangdong, China; ${ }^{2}$ University of North Carolina Project-China, China

\subsection{6/sextrans-2021-sti.357}

Background The overuse of antibiotics accelerates antibiotic resistance. However, limited studies assessed the antibiotic use history and its association with current chlamydia infection among women of reproductive age in China. This study aimed to investigate the recent antibiotic usage pattern and its association with current chlamydia infection among reproductiveaged women (RAW) in gynaecology clinics in Guangdong, China.

Methods We conducted a cross-sectional study to recruit women aged between 16 to 55 in Guangdong. All participants were tested for chlamydia by using Nucleic Acid Amplification Testing and finished a computer-based survey containing their self-reported antibiotic consumption in recent 12 months. We described characteristics of participants and recent antibiotic usage patterns based on two different classifications at first. Then we calculated the antibiotics usage rate and analyzed the 